\title{
Performance of the SASE amplifier of the TEU-FEL project
}

\author{
G.J. Ernst and J.C. Goldstein ${ }^{1}$ \\ Unic ersity of Twente, Department of Applied Physics, P.O. Box 217, 7500 AE Enschede, The Netherlands,
}

The free-electron laser of the TEU-FEL project of the University of Twente will be driven by a photoinjector followed by a racetrack microtron. The injector, which is now under construction, will provide a very high-brightness electron beam with an energy of about $6 \mathrm{MeV}$. In phase I of the project, experiments are being planned in which this low energy beam from the injector will pass through an undulator and will generate radiation at a wavelength of about $200 \mu \mathrm{m}$ via the process of self-amplified spontaneous emission (SASE). Numerical simulations of the performance of this source indicate that power levels of about $15 \mathrm{MW}$ (averaged over a micropulse) can be obtained with a $1-\mathrm{m}$ undulator. We present additional results derived from simulation studies of the performance of this device.

\section{Introduction}

The name TEU-FEL is an acronym for Twente/ Eindhoven University UCN Free-Electron Laser [1]. It will be a pulsed FEL oscillator driven by a $25 \mathrm{MeV}$ electron beam of moderate to high peak current [2]. The electron source will consist of a photocathode injector/rf-linac which is presently being built at Los Alamos National Laboratory. It will be shipped in the near future to The Netherlands. The injector linac will generate a high-brightness ( $\sim 350$ A peak current, "90\%" normalized transverse emittance $\epsilon_{n}=25 \pi \mathrm{mm}$ mrad), low-energy ( $\sim 6 \mathrm{MeV})$ beam which will be injected into a 1-m wiggler [3] in phase $I$ of the planned FEL experiments. The phase-I experiments will study high-gain self-amplified spontaneous emission (SASE) [4] FEL physics near an optical wavelength of $\lambda \approx 200$ $\mu \mathrm{m}$. Oscillator experiments at $\lambda \approx 10 \mu \mathrm{m}$ will constitute the second phase of the experimental program upon completion of the racetrack microtron. In the present work, we present some results of numerical simulation studies of the high-gain FEL physics for the phase-I SASE amplifier experiments. Both $1 D$ and 3D FEL simulation codes [5-7] were used to study the properties of this device. A high-gain FEL amplifier is expected [8] to exhibit strong optical guiding effects when the light intensity is low enough to be within the small-signal regime. A key parameter is the small-signal power e-folding length $z_{\mathrm{g}}$, where the total pówer $P$

' Permanent address: Group X-1, MS F645, Los Alamos National Laboratory, Los Alamos, NM 87544, USA.

* Sponsored by Nederlands Centrum voor Laser Research (NCLR), the Dutch Foundation for Technological Research (STW), and the Dutch Foundation for Research on Matter (FOM). of the guided mode varies with distance $z$ along the wiggler's axis according to $P(z)=P_{0} \exp \left(z / z_{\mathrm{g}}\right)$. We have computed the gain length $z_{\mathrm{g}}$ for the phase-I experiments. When the gain starts to saturate, optical guiding is lost and the mode's transverse profile changes. We have observed these changes as a function of wiggler length in 3D FEL simulations. Finite-pulse 3D simulations were used to predict the SASE output. In these experiments, the slippage distance $s(s=N \lambda$, where $N$ is the number of periods of the wiggler, $L_{\mathrm{w}}=N \lambda_{\mathrm{w}}$ ) will be approximately equal to the electron pulse length; hel.ce, slippage effects are important. In section 2 we present the expected parameter values for the phase-I experiments which were used in the numerical simulations. The results of the simulations are presented in section 3, while a summary and a discussion of the results is given in section 4 .

\section{Expected parameter values for the phase-I experi- ments}

Table 1 summarizes the values of the wiggler and electron beam parameters which were used in the simulations. The wiggler [3] will have a $2.5 \mathrm{~cm}$ period and a $K_{\mathrm{rms}}$ value equal to one $\left(a_{\mathrm{w}}=\sqrt{2}\right)$. It will have two-plane focusing with equal focal strengths in the two transverse directions. The full gap between the poles will be $0.8 \mathrm{~cm}$, but a beam tube of inner diameter $0.6 \mathrm{~cm}$ will be used to transport both the electron and optical beams through the wiggler. The nominal length of the wiggler will be $1 \mathrm{~m}$, and quadrupoles will be used to match the electron beam into the wiggler.

The electron beam is assumed to have an energy of $6 \mathrm{MeV}\left(\gamma_{0}=11.74\right)$, a "90\%" normalized transverse emittance of $50 \pi \mathrm{mm}$ mrad. a fractional energy spread 
Table 1

The main parameters of the TEU-FEL phase-I experiments

\begin{tabular}{|c|c|}
\hline \multicolumn{2}{|l|}{ Undulator parameter ialues: } \\
\hline Wavelength $\lambda_{w}$ & $2.5 \mathrm{~cm}$ \\
\hline Length $L_{w}$ & $100 \mathrm{~cm}$ \\
\hline Number of periods $N=L_{w} / \lambda_{w}$ & 40 \\
\hline Peak magnetic field amplitude $B_{w}$ & $6064.535 \mathrm{G}$ \\
\hline \multirow{2}{*}{\multicolumn{2}{|c|}{$\begin{array}{l}\text { Dimensionless vector potential } a_{w} \\
\text { Two-plane focusing with equal strength in both planes }\end{array}$}} \\
\hline & \\
\hline Electron beam parameter calues: Energy $E$ & $6 \mathrm{MeV}$ \\
\hline$E / m c^{2}=\gamma$ & 11.74 \\
\hline $\begin{array}{l}90 \% \text { normalized transverse emittance } \epsilon_{\mathrm{\pi}} \\
\text { Gaussian phase-space density }\end{array}$ & $50 \pi \mathrm{mm}$ mrad \\
\hline Fractional energy spread (FWHM) $\Delta y / \gamma$ & $1 \%$ \\
\hline Peak current $I$ & $350 \mathrm{~A}$ \\
\hline Pulse length tc & $35 \mathrm{ps}$ \\
\hline \multicolumn{2}{|l|}{ Optical parameter ralues: } \\
\hline Resonance wavelength $\lambda$, & $181.33 \mu \mathrm{m}$ \\
\hline Slippage distance $s=N \lambda$, & $24.178 \mathrm{ps}$ \\
\hline
\end{tabular}

(FWHM) of $\Delta y / \gamma_{0}=1 \%$, and a peak current of $I=$ 350 A. These are conservative values: we expect the operational linac to achieve one-half of the emittance value assumed here, and the energy spread will probably be one-third of the value assumed here. We expect the electron energy to be variable between 3 and 8 MeV. We have assumed a Gaussian phase-space density for the beam in the simulation model. The simulations have been done for an electron beam micropulse length of $35 \mathrm{ps}$. However, the electron pulse length will be adjustible down to a few ps by varying the length of the optical pulse from the photocathode drive laser. For the above wiggler and electron beam parameters, the resonance optical wavelength is $\lambda_{\mathrm{s}}=181.33 \mu \mathrm{m}$,

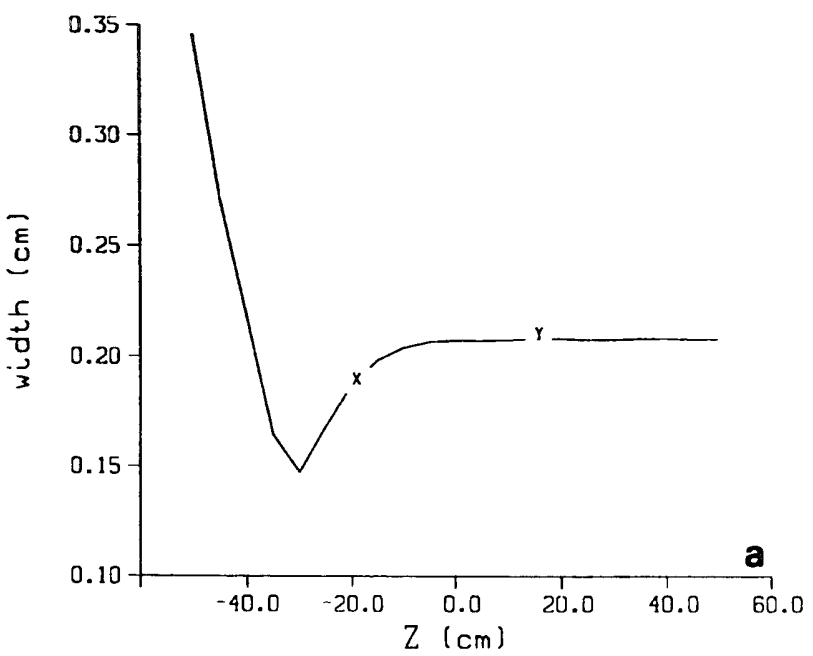

and the slippage distance $s=N \lambda_{\mathrm{s}}$ corresponds to 24.178 ps.

\section{Numerical simulations of the phase-I experiments}

All simulations assume that the FEL will operate in the Compton regime in which space-charge effects are ignored. For the parameter values of table 1 , the radius of the electron beam in the wiggler is $3.75 \times 10^{-2} \mathrm{~cm}$, and the electron number density on axis is $1.65 \times 10^{13}$ $\mathrm{cm}^{-3}$. Using these values, we determine that the ratio of the longitudinal plasma frequency in the lab frame, $\omega_{\mathrm{pe}} / \gamma^{3 / 2}$, to the frequency of the electromagnetic wave, $\omega_{\mathrm{em}}=2 \pi c / \lambda_{\mathrm{s}}$, is about $5.5 \times 10^{-4}$. This puts the device squarely in the Compton regime, and, furthermore, we find that the transit time of the electron pulse through a 1-m wiggler corresponds to about three plasma periods.

\subsection{Calculation of the optical power e-folding length $z_{g}$}

The power e-folding length was calculated for a continuous electron beam (pulse effects were ignored) by injecting a very low intensity coherent signal at a fixed wavelength into the amplifier. Since the overall gain of the device is very large $\left(\approx 10^{10}\right)$, an unphysically small initial optical intensity (less than the spontaneous emission from a short length of wiggler) is needed in order that the light remains in the small-signal regime (where the gain is independent of the amplitude of the initial input signal) throughout the wiggler. In one dimension, the optical power does not grow exponentially with distance [9] until the growing mode dominates the other modes (one with a decaying amplitude and one with an oscillating amplitude) which are

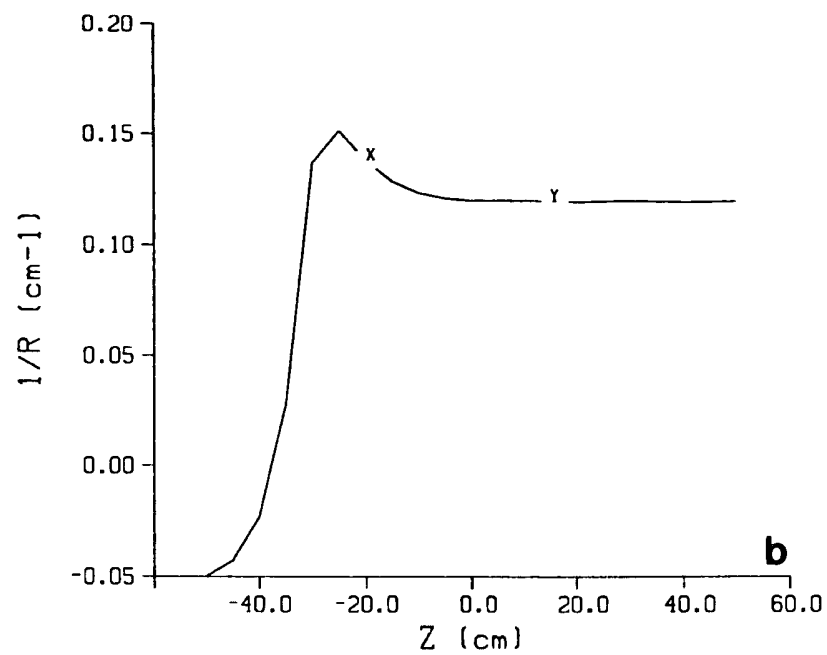

Fig. 1. (a) Radius of optical beam vs axial position for the highest-gain mode. (b) Reciprocal of the radius of curvature vs. axial position for the highest-gain mode. 
Table 2

Variation with current and emittance of guided mode properties from 3D simulations

\begin{tabular}{lcllc}
\hline$I[\mathrm{~A}]$ & $\epsilon_{\mathrm{n}}[\pi \mathrm{mm} \mathrm{mrad}]$ & $z_{\mathrm{g}}[\mathrm{cm}]$ & $w[\mathrm{~cm}]$ & $R[\mathrm{~cm}]$ \\
\hline 350.0 & 50.0 & 3.88 & 0.207 & 8.31 \\
350.0 & 100.0 & 4.35 & 0.221 & 9.70 \\
262.5 & 50.0 & 4.44 & 0.227 & 9.38 \\
262.5 & 100.0 & 4.98 & 0.241 & 10.92 \\
175.0 & 50.0 & 5.37 & 0.247 & 11.36 \\
175.0 & 100.0 & 6.07 & 0.276 & 12.99 \\
\hline
\end{tabular}

excited at the entrance to the wiggler. In three dimensions, a similar mode competition effect occurs in which the optical power does not grow exponentially until the highest-gain guided mode becomes dominant. Since one does not know analytically the mode spot size and curvature of the dominant mode, the injected signal is initially multitransverse mode; some distance of propagation is required until one mode dominates.
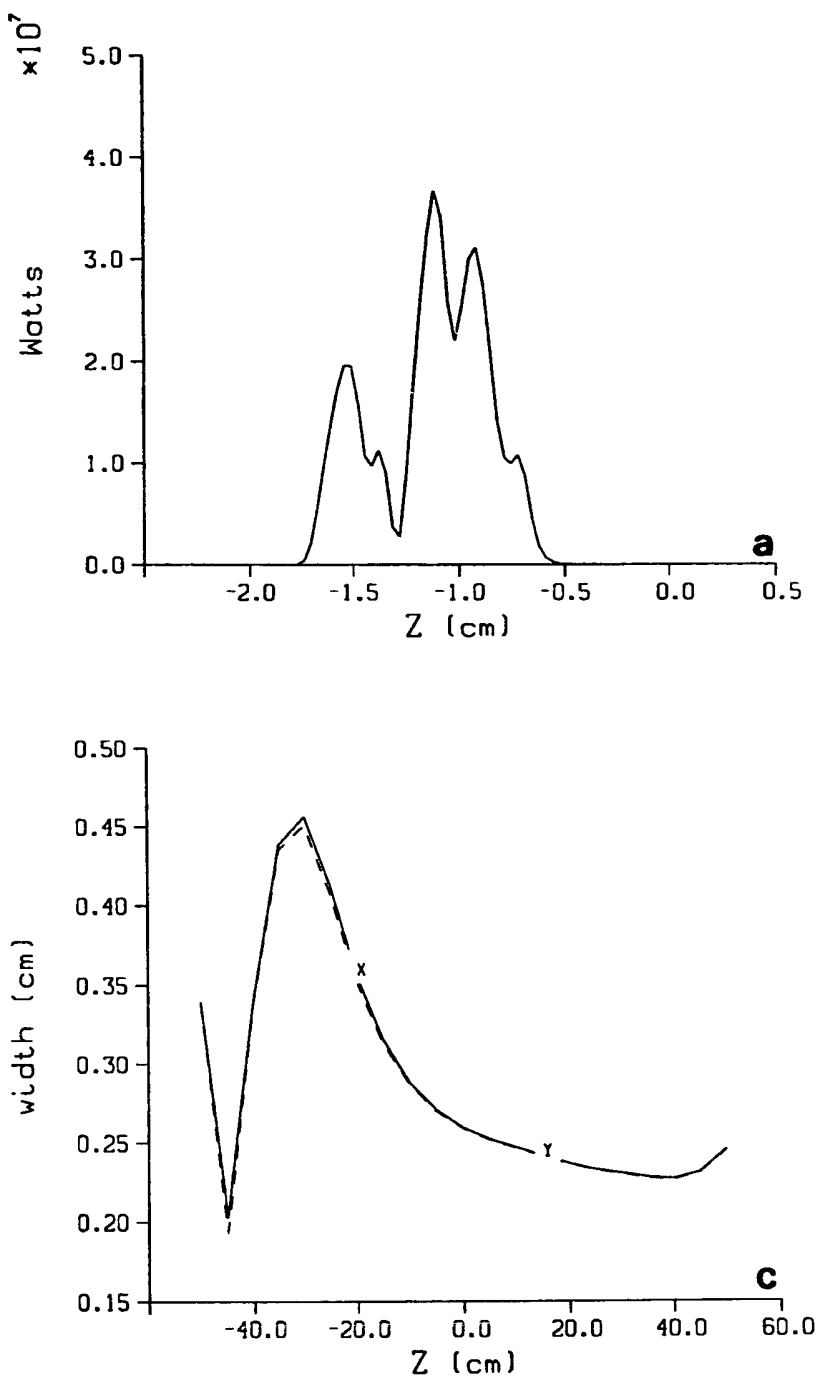

In all cases, quoted values of $z_{\mathrm{g}}$ are obtained after multimode transients have vanished, leaving a single mode with an exponentially-growing amplitude.

1D simulations with the parameters of table 1 give a minimum (over optical wavelength) value of $z_{g}=1.9$ $\mathrm{cm}$ at $\lambda=189 \mu \mathrm{m}$; this is in good agreement with the formula [4] $z_{\mathrm{g}}=\lambda_{\mathrm{w}} /(8 \pi \mu \rho)$ which gives $z_{\mathrm{g}}=2.0 \mathrm{~cm}$. Here, $\mu=0.5 \sqrt{3}$ in one dimension, and $\rho$ is the dimensionless FEL parameter (sometimes called the Pierce parameter) introduced in 1984 by Bonifacio, Pellegrini, and Narducci [4].

3D single-wavelength simulations give a minimum value of $z_{\mathrm{g}}=3.88 \mathrm{~cm}$ at $\lambda=188 \mu \mathrm{m}$. Figs. $1 \mathrm{a}$ and $\mathrm{lb}$ show how the mode's radius and wavefront curvature evolve along the wiggler's length in the simulation. The mode radius $w$ (which is called the width in fig. 1a) is defined to be $w=\sqrt{2} \times$ rms radius; if the transverse profile of the guided mode were Gaussian (which it is not), this would equal the radius at the $1 / \mathrm{e}$ point of the electric field amplitude.
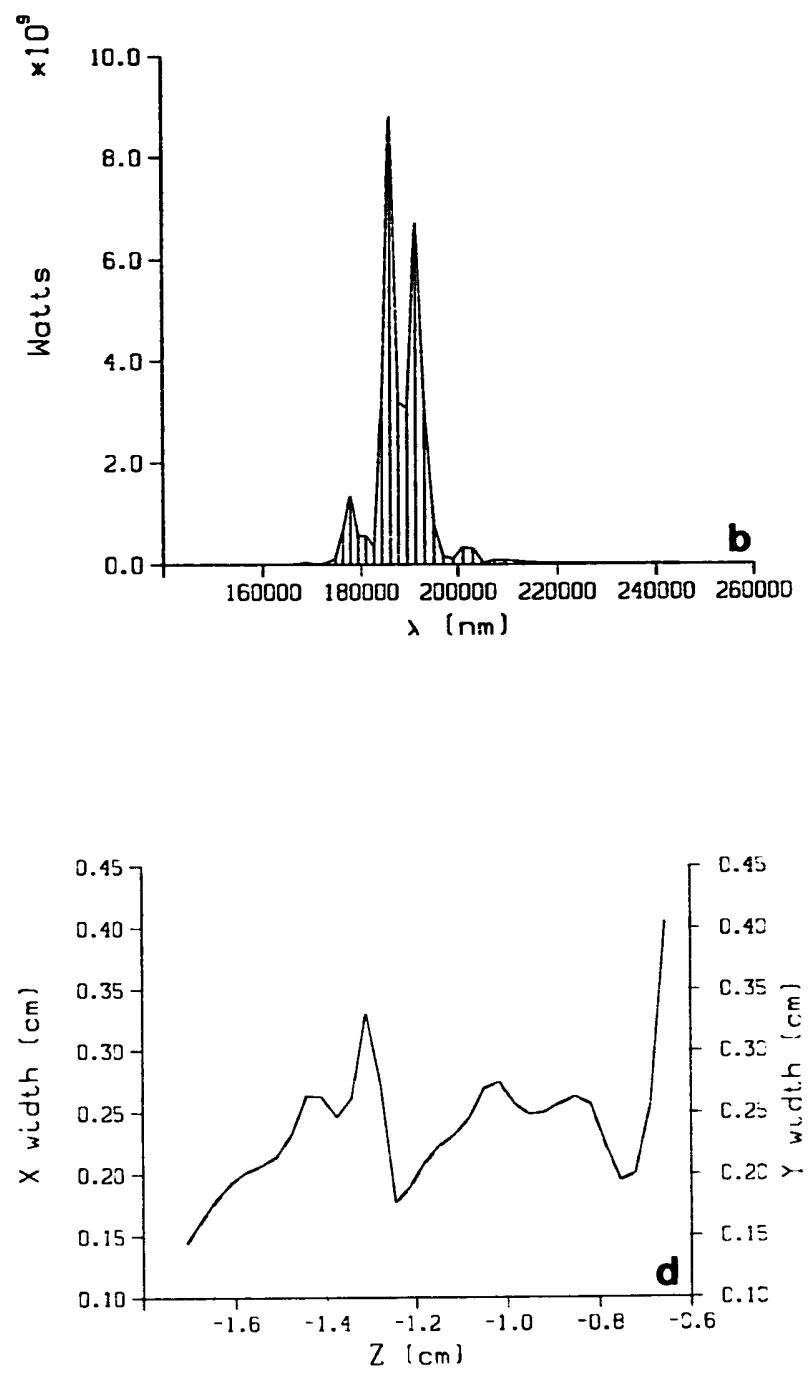

Fig. 2. (a) Optical pulse s.rape at the wiggler exit. (b) Optical spectrum at the wiggler exit (pass number $=1$ ). (c) Mean radius vs axial position. (d) Radius vs position within micropulse at wiggler exit (pass number $=1$ ). 
Table 2 shows the results of a series of 3D simulations with varying values of the peak current in amperes $I$ and the " $90 \%$ " normalized transverse emittance $\epsilon_{\mathrm{n}}$. In table $2, z_{\mathrm{g}}$ is the power e-folding length, $w$ is the mode radius, and $R$ is the mode radius of curvature. A positive value of $R$ indicates a diverging wavefront. All quoted values are obtained after the initial transients have subsided and a steady-state guided mode has been achieved.

\subsection{Results of 3-D finite-pulse simulations of SASE performance}

3D finite-pulse simulations of the SASE performance of the phase-I TEU-FEL experiments were done with the FEL simulation code FELEX [5-7]. The electron pulse was assumed to be rectangular in shape (constant current vs time). Full slippage between the light and the electron pulses is included in the simulations. The initial radiation results from spontaneous
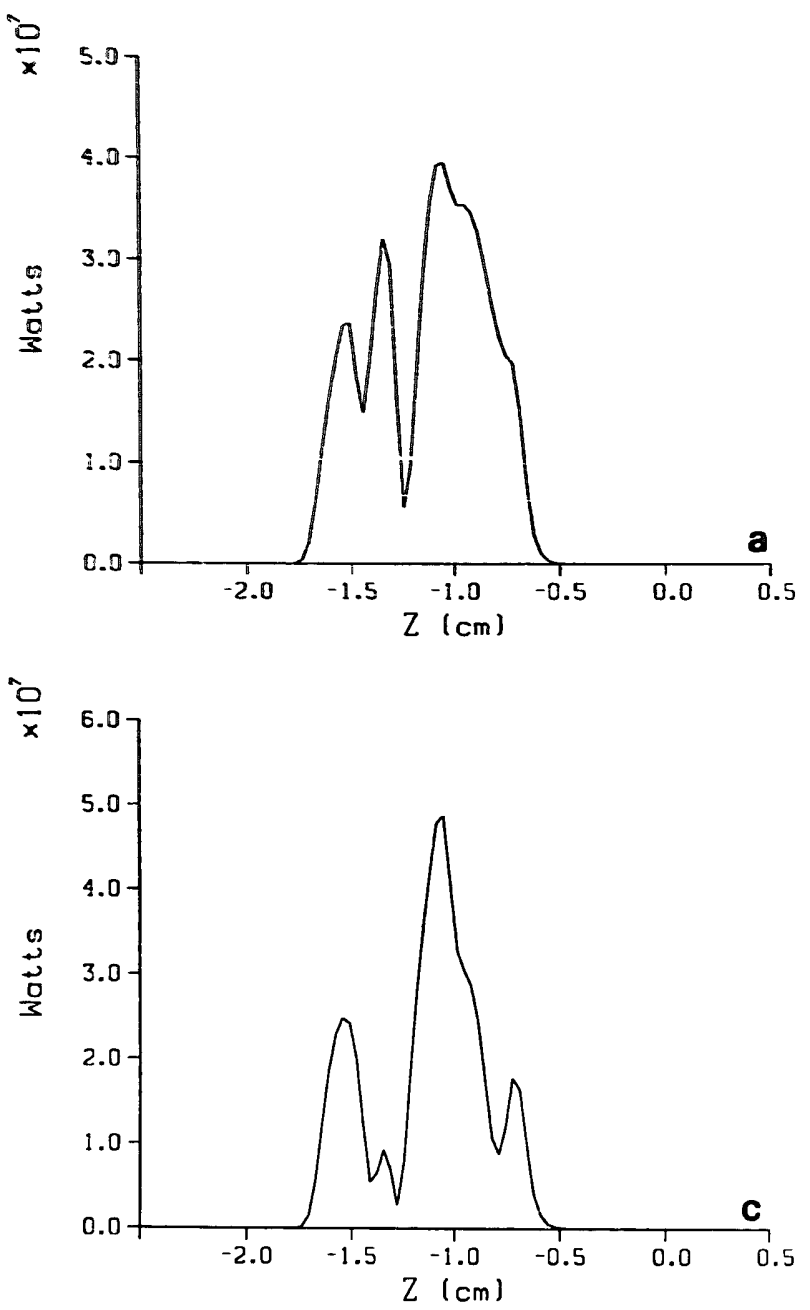

emission [10] in the initial segments of the wiggler rather than an injected external coherent signal.

For the parameters of this experiment, we find that the shape and amplitude of the optical pulse at the end of the wiggler depend on the specific form of the initial spontaneous emission noise. Due to the relatively small number of simulation electrons in the calculations, the fluctuations of the numerically-generated spontaneous emission are unphysically large. It is necessary to repeat the simulation with different initial random number seeds (which produce different realizations of the spontaneous emission) and then average the results in order to predict the mean results of an experiment.

The mathematical model in the code assumes freespace propagation of the electromagnetic field. However, in the experiments of phase I an evacuated tube $0.6 \mathrm{~cm}$ in diameter will be placed within the gap of the wiggler. Both the electrons and the light will pass through this tube. We have not included in the simulations effects due to reflections of light from the tube walls, or waveguide mode effects.
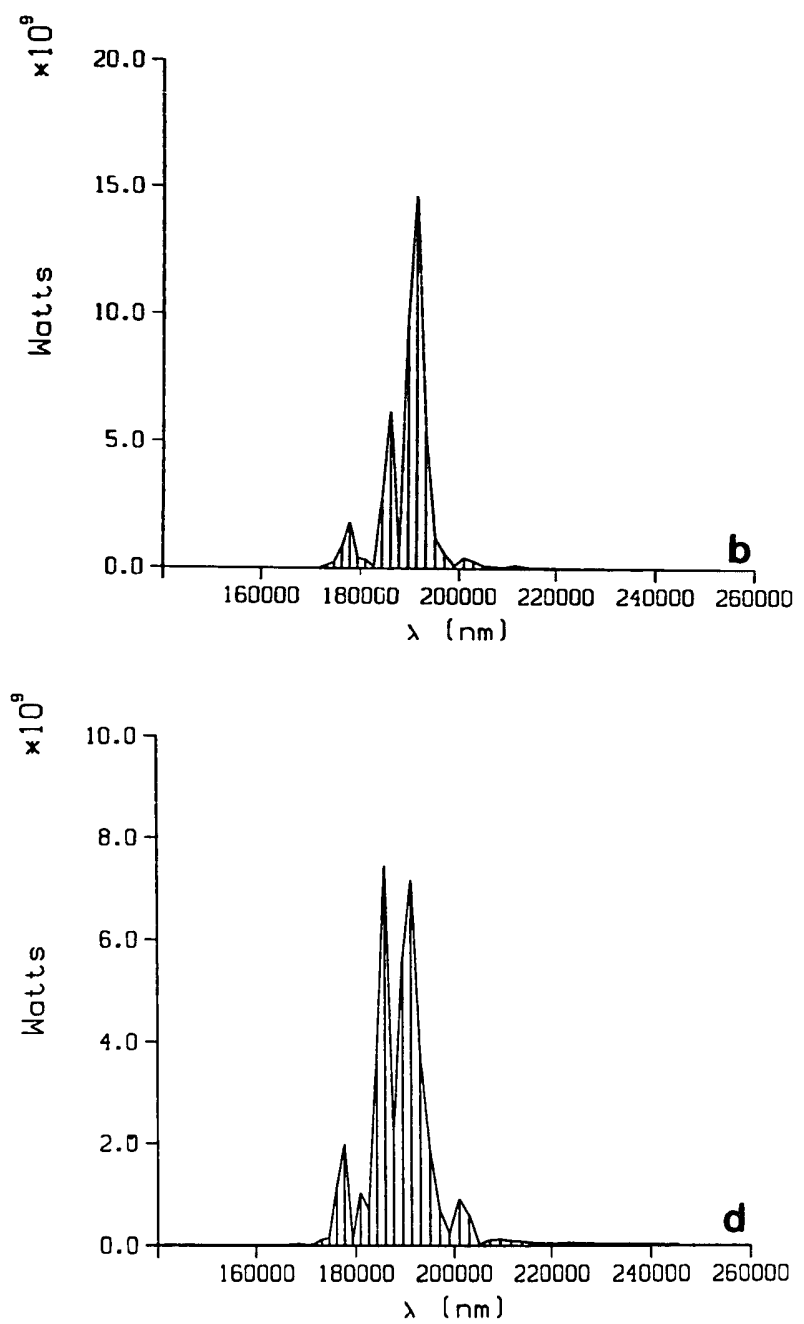

Fig. 3. (a) Optical pulse shape for initial seed (1). (h) Optical spectrum for (1) (pass number $=1$ ). (c) Optical pulse shape for initial seed 92) (pass number $=1$ ) (d) Optical spectrum for (2). (pass number $=1$ ). 
Table 3

SASE results from 3D finite-pulse simulations

\begin{tabular}{lclll}
\hline$I[\mathrm{~A}]$ & $\epsilon_{\mathrm{n}}[\pi \mathrm{mm}-\mathrm{mr}]$ & $\eta[\%]$ & $E_{\text {light }}[\mathrm{mJ}]$ & Power $[\mathrm{MW}]$ \\
\hline 350.0 & 50.0 & 1.0 & 0.8 & 24.0 \\
350.0 & 100.0 & 0.14 & 0.1 & 3.0 \\
262.5 & 50.0 & 0.1 & 0.06 & 3.6 \\
175.0 & 50.0 & 0.003 & 0.001 & 0.03 \\
\hline
\end{tabular}

Fig. 2 shows typical results for the SASE performance of the reference case (parameters of table 1). Fig. 2a shows the optical micropulse at the end of the wiggler, fig. $2 \mathrm{~b}$ shows the spectrum, fig. $2 \mathrm{c}$ shows the axial variation of the radius of the light (averaged over the optical micropulse), and fig. $2 \mathrm{~d}$ shows the variation of the optical radius within the micropulse (whose power variation is shown in fig. 2a) at the end of the wiggler.

Fig. 3 shows the results of two simulations identical in all respects with that which yielded the results of fig. 2 except that the initial random number seed of the spontaneous emission noise was changed. Figs. 3a and $3 b$ show the output power micropulse and corresponding spectrum for one seed, and figs. $3 c$ and $3 d$ show similar curves for another seed.

Table 3 shows a summary of SASE results from 3D finite-pulse simulations for a variation of the peak current $I$ and the "90\%" normalized transverse emittance $\epsilon_{\mathrm{n}}$. In table $3, \eta$ is the pulse-averaged extraction efficiency, $E_{\text {light }}$ is the total energy of the optical pulse, and "power" is the observed peak power of the optical micropulse. The tabulated values represent averaged over several calculations with different initial random number seeds; an approximate spread of $\pm 20 \%$ should be associated with each average value to account for results with different initial random number seeds.
Increasing the length of the wiggler to $L_{w}=125 \mathrm{~cm}$ approximately doubles the output optical power relative to that produced by an undulator of length $L_{w}=$ $100 \mathrm{~cm}$ (for parameters of the reference case). However, the gain starts to saturate over the last $25 \mathrm{~cm}$ of the wiggler, and the mode radius grows significantly larger than the radius of the beam tube $(0.3 \mathrm{~cm})$. This is shown in fig. 4: the output power pulse is shown in fig. 4a while the corresponding axial variation of the average optical mode radius is shown in fig. $4 \mathrm{~b}$. Since wall reflections are not included in the simulations, it is not clear just what modification of these results would occur due to such reflections.

\section{Summary and discussion}

We have studied the performance of the $\lambda \sim 200$ $\mu \mathrm{m}$ SASE amplifier of the phase-I experiments of the TEU-FEL project by means of $1 D$ and 3D numerical simulations. The 1D small-signal power e-folding length $(\sim 2 \mathrm{~cm})$ considerably underestimates the gain length according to the 3D simulations which give a value of about $3.9 \mathrm{~cm}$. We have studied the dependence of the gain length on the peak current and transverse emittance for values about those of the reference design. Note that we cannot directly apply the results of a recent quasi-analytical calculation [11] for the gain length because those results hold for a uniformly-filled phase-space distribution ("water bag") and not a Gaussian phase-space density which we have assumed.

We have calculated the characteristics of the optical pulses produced by the self-amplified spontaneous emission process. For a 1-m wiggler, it appears likely that peak powers in excess of $20 \mathrm{MW}$ will be produced along with an extraction efficiency of about $1 \%$ if the design parameters are achieved experimentally. We

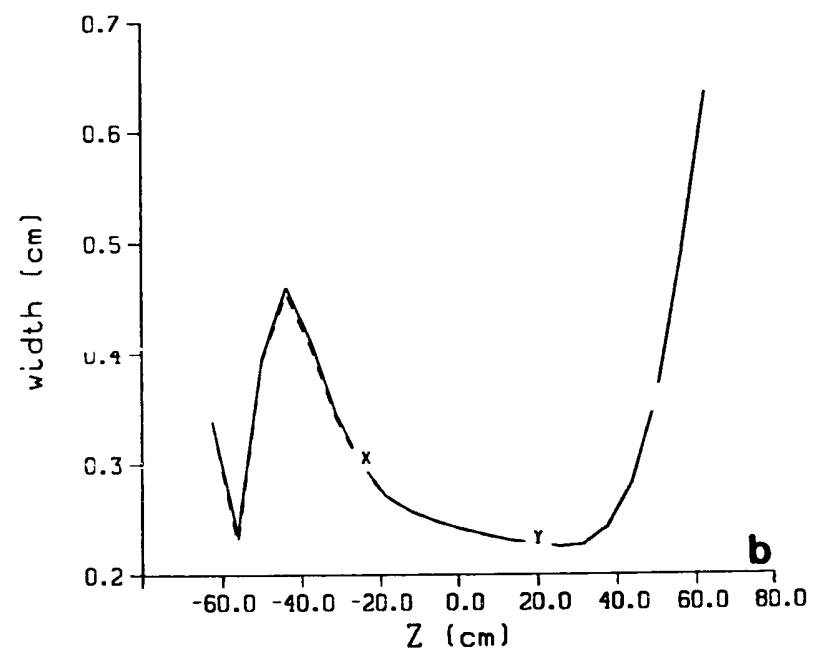

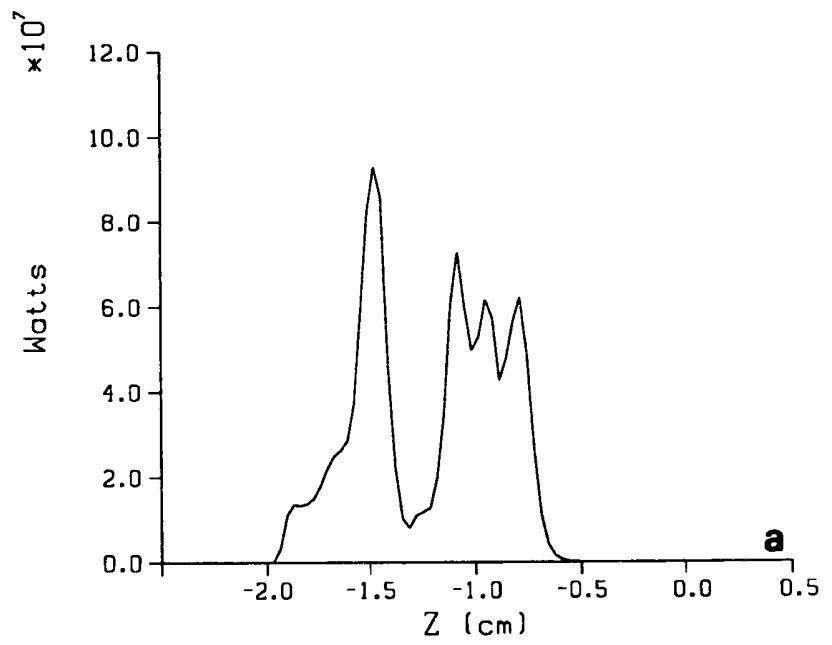

Fig. 4. (a) Optical pulse shape for $L_{w}=125 \mathrm{~cm}$. (pass number $=1$ ). (b) Mean radius vs axial position for $L_{\mathrm{w}}=125 \mathrm{~cm}$. 
have calculated the expected variation of the optical radiation for various values of peak current and emittance.

Lengthening the wiggler increases the output power, but also increases the radius of the transverse mode (due to gain saturation) such that the light will be reflected from the walls of the beam tube which passes through the wiggler's bore. This will certainly reduce the optical beam quality of the emerging radiation pulses.

Finally, some preliminary simulations show that measurable amounts of radiation in higher harmonics may be produced in these experiments. Measurements of such radiation may add to the understanding of such high-gain amplifiers.

\section{Acknowledgement}

The authors would like to thank T.J.T. Kwan for helpful discussions related to the possible Raman-regime character of this system.

\section{References}

[1] G.J. Ernst, W.J. Witteman, J.W.J. Verschuur, R.F.X.A.M. Mols, E.H. Haselhoff, J.I.M. Botman, J.L. Delhez, H.L. Hagedorn and W.J.G.M. Kleeven, these Proceedings (13th Int. Free Electron Laser Conf., Santa Fe, USA, 1991) Nucl. Instr. and Meth. A318 (1992) 173.

[2] J.I.M. Botman, J.L. Delhez, G.A. Webers, H.L. Hagedoorn, W.J.G.M. Kleeven, C.J. Timmermans, G.J. Ernst, J.W.J. Verschuur, W.J. Witteman and E.H. Haselhoff, Nucl. Instr. and Meth. A304 (1991) 192.

[3] J.W.J. Verschuur, G.J. Ernst and W.J. Witteman, these Proceedings (13th Int. Free Electron Laser Conf., Santa Fe, USA, 1991) Nucl. Instr. and Meth. A318 (1992) 847.

[4] K.J. Ki, Phys. Rev. Lett. 57 (1986) 1871.

[5] B.D. McV.y, Nucl. Instr. and Meth. A250 (1986) 449.

[6] B.D. McVey, J.C. Goldstein, R.L. Tokar, C.J. Elliott, S.J. Gitomer, M.J. Schmitt and L.E. Thode, Nucl. Instr. and Meth. A285 (1989) 186.

[7] J.C. Goldstein, B.D. McVey, R.L. Tokar, C.J. Elliott, M.J. Schmitt, B.E. Carlsten and L.E. Thode, in: Modeling and Simulation of Laser Systems, ed. D.L. Bullock, Proc. SPIE 1045 (1989) 28.

[8] G.T. Moore, Nucl. Instr. and Meth. A250 (1986) 381.

[9] C.C. Shih and A. Yariv, IEEE J. Quant. Electron. QE-17 (1981) 1387.

[10] M.J. Schmitt, Proc. Conf. on Short Wavelength Radiation Sources, San Diego, CA, July 24-25, 1991.

[11] L.H. Yu, S. Krinsky, and R.L. Gluckstern, Phys. Rev. Lett. 64 (1990) 3011. 